\title{
Prediction of the Black-Hole Mass in 3C 273 by Multiband Observations
}

\author{
Zhen Guo Ma and Xi Zhen Zhang \\ National Astronomical Observatories, Chinese Academy of Sciences, \\ Beijing, 100012, China
}

\begin{abstract}
With the determined black-hole (BH) spin of $3 \mathrm{C} 273$ by data-fitting to the detected iron $\mathrm{K} \alpha$ line emission in the soft $\mathrm{X}$-ray band, the $\mathrm{BH}$ mass of the galaxy is predicted by formulations of both the observed disk-luminosity in the optical-UV band and the observed jetprecession in the radio band. The multiband synthesis suggests that the $\mathrm{BH}$ is supermassive, $2.4 \times 10^{9} M_{\odot}$. Simultaneously, other physical parameters are self-consistently obtained at the precessing radius of $230.2 r_{g}$ : the accretion rate of the disk is $74.9 M_{\odot} \mathrm{yr}^{-1}$, the Shakura-Sunyaev viscosity $\alpha$ is 0.134 , and the radial \& orbital velocities of fluid elements are $4.3 \times 10^{-8}$ and $6.6 \times 10^{-2}$, respectively.
\end{abstract}

\section{Introduction}

The nearby quasar 3C 273 is one of the most extensively observed extragalactic objects. Besides its high luminosity over the whole bands (Leach, McHardy, \& Papadakis 1995), many other features were also found, such as, the jet-precession period is $1.5 \times 10^{4}$ years (Davis, Muxlow, \& Conway 1985), the mass inflow rate of the galaxy is $100 M_{\odot} \mathrm{yr}^{-1}$ (Roos 1988), and the Seyfert-like $\mathrm{Fe} \mathrm{K} \alpha$ emission is $5.4 \times 10^{43} \mathrm{ergs} \mathrm{s}^{-1}$ at $6.4 \mathrm{keV}$ (Yaqoob \& Serlemitsos 2000).

However, the mass of the central black-hole $(\mathrm{BH}), M_{\mathrm{BH}}$, in $3 \mathrm{C} 273$ has been suggested full of variety from $10^{8} M_{\odot}$ to $10^{11} M_{\odot}$ with different methods (Kafatos 1980; Malkan 1983; Wandel \& Mushotzky 1986; Dermer \& Gehrels 1995; Hayashida et al. 1998; Laor 1998; Kriss et al. 1999; Romero et al. 2000; Czerny et al. 2001). This paper develops a new approach to estimate $M_{\mathrm{BH}}$ of 3C 273 within a synthesized framework of previous multiband observations and theoretical models in the soft-X ray, optical-UV, and radio bands.

\section{Prediction of the BH spin with X-ray Fe line observation}

In the soft-X ray band, the detected iron $\mathrm{K} \alpha$ line comes from the fluorescence of matter within $20 r_{g}\left(r_{g}=G M_{\mathrm{BH}} / c^{2}\right)$ in a plane from the central BH (Fabian et al. 2000), providing information about the BH spin, emitter's radial position, and the observer's inclination angle (Ma 2002). The predicted parameters are: (1) the BH spin is $a=0.94 \pm 0.005$; (2) the radius of the thin-torus emitting Fe $\mathrm{K}$ line is $r=4.0 \pm 0.05 r_{g}$; (3) the inclination angle of the observer relative 
to the $\mathrm{BH}$ axis is $\theta_{0}=29 \pm 0.5^{\circ}$ and thus the angle to the equatorial disk is $\theta_{0}=61 \pm 0.5^{\circ}$.

\section{Prediction of the disk accretion rate with optical-UV luminosity observation}

In the optical-UV band, the observed spectrum [for 3C 273 the luminosity is $\sim 3 \times 10^{47} \mathrm{ergs} \mathrm{s}^{-1}$ (Courvoisier \& Camenzind 1989)] is found to be bestfitted with the standard thin-disk black-body (BB) thermal emission with PageThorne (PT) function (Page \& Thorne 1974).

If not specifically noted, we choose natural units $G=c=1$ and a geometrized radial coordinate $r$ in the unit of $r_{g}=M_{\mathrm{BH}}$, the gravitational radius of the BH. If $a=J / M_{\mathrm{BH}}^{2}$ denotes the dimensionless specific angular momentum of the $\mathrm{BH}$ with the angular momentum $J$, the dimensionless radius of the marginally stable circular orbit, $r_{m s}$, in the equatorial plane is (Bardeen, Press, \& Teukolsky 1972): $r_{m s}=3+Z_{2} \mp\left[\left(3-Z_{1}\right)\left(3+Z_{1}+2 Z_{2}\right)\right]^{1 / 2}, Z_{1}=$ $1+(1-a)^{1 / 3}\left[(1+a)^{1 / 3}+(1-a)^{1 / 3}\right], \quad Z_{2}=\left(Z_{1}^{2}+3 a^{2}\right)^{1 / 2}$ where the upper and lower signs are for the cases of the prograde disk (i.e., rotating in the same direction as the $\mathrm{BH}$ ) and the retrograde one, respectively.

The relation between the bolometric luminosity of the standard disk, $L_{\text {disk }}$, and the local effective BB temperature $T(r)$ at $r$ takes the form (e.g., Makishima et al. 1986): $L_{\text {disk }}=\int_{r_{\text {in }}}^{r_{\text {out }}} 4 \pi r \sigma T^{4}(r) d r$ in which $\sigma$ is the Stefan-Boltzmann constant, $r_{\text {in }}$ and $r_{\text {out }}$ are respectively the inner and outer boundaries of the disk. In the expression, $T(r)$ is shown by PT function: $T(x)=T_{0}[f(x)]^{1 / 4}$, $f(x)=f_{0}(x)\left[x-x_{0}-f_{1}(x)-f_{2}(x)-f_{3}(x)-f_{4}(x)\right]^{1 / 4}$ is the general relativistic (GR) expression of the PT gravitational energy release per unit area from the disk surface as a function of $a$, in which $f_{0}(x)=\left[x^{4}\left(x^{3}-3 x+2 a\right)\right]^{-1}$, $f_{1}(x)=1.5 a \ln \left(\mathrm{x} / \mathrm{x}_{0}\right), f_{2}(x)=3\left(x_{1}-a\right)^{2} \ln \frac{\left(\mathrm{x}-\mathrm{x}_{1}\right) /\left(\mathrm{x}_{0}-\mathrm{x}_{1}\right)}{\mathrm{x}_{1}\left(\mathrm{x}_{1}-\mathrm{x}_{2}\right)\left(\mathrm{x}_{1}-\mathrm{x}_{3}\right)}, f_{3}(x)=3\left(x_{2}-\right.$ $a)^{2} \ln \frac{\left(\mathrm{x}-\mathrm{x}_{2}\right) /\left(\mathrm{x}_{0}-\mathrm{x}_{2}\right)}{\mathrm{x}_{2}\left(\mathrm{x}_{2}-\mathrm{x}_{1}\right)\left(\mathrm{x}_{2}-\mathrm{x}_{3}\right)}, f_{4}(x)=3\left(x_{3}-a\right)^{2} \ln \frac{\left(\mathrm{x}-\mathrm{x}_{3}\right) /\left(\mathrm{x}_{0}-\mathrm{x}_{3}\right)}{\mathrm{x}_{3}\left(\mathrm{x}_{3}-\mathrm{x}_{1}\right)\left(\mathrm{x}_{3}-\mathrm{x}_{2}\right)}$, and $x_{0}=r_{m s}^{1 / 2}, x_{1}=$ $2 \cos \left(\cos ^{-1} \frac{\mathrm{a}}{3}-\frac{\pi}{3}\right), x_{2}=2 \cos \left(\cos ^{-1} \frac{\mathrm{a}}{3}+\frac{\pi}{3}\right), x_{3}=-2 \cos \left(\cos ^{-1} \frac{\mathrm{a}}{3}\right)$ where $x=r^{1 / 2}$; $T_{0} \equiv\left(3 \dot{M} c^{6}\right)^{1 / 4}\left(8 \pi \sigma M_{\mathrm{BH}}^{2} G^{2}\right)^{-1 / 4}$ is the characteristic temperature (cgs units) in which $\dot{M}$ is the mass accretion rate.

Define $m=\frac{M_{\mathrm{BH}}}{10^{9} M_{\odot}}, l=\frac{L_{\mathrm{disk}}}{L_{\mathrm{Edd}(m=1)}}$, and $\dot{m}=\frac{\dot{M}}{10 \dot{M}_{\mathrm{Edd}(m=1)}}$, in which $L_{\operatorname{Edd}(m=1)}$ and $\dot{M}_{\mathrm{Edd}(m=1)}$ are the values of the Eddington luminosity and accretion rate, respectively, when $m=1: L_{\mathrm{Edd}}=1.2580 \times 10^{47} \mathrm{~m} \mathrm{ergs} \mathrm{s}^{-1}, \dot{M}_{\mathrm{Edd}}=\frac{L_{\mathrm{Edd}}}{c^{2}}=$ $2.2916 m M_{\odot} /$ yr. Then,

$$
\frac{l}{\dot{m}}=30 \int_{r_{\mathrm{in}} 1 / 2}^{r_{\mathrm{out}}{ }^{1 / 2}} x^{3} f(x) d x
$$

which shows that $l$ is connected to both $\dot{m}$ and $a$ but not $m$. In calculations, $r_{i n}=r_{m s}$. For $r_{\text {out }}>2500$, the ratio $\frac{l}{\dot{m}}$ versus the BH spin $a$ is equal to the the value at $r_{\text {out }}=2500$. Thus, we select $r_{\text {out }}=2500$. If $l$ and $a$ are known, the mass accretion rate $\dot{m}$ can then be determined.

However, the observed optical-UV luminosity cannot be used directly. There is a simple relation between the observed luminosity $L_{\text {obs }}$ and the bolometric 
one $L_{\text {disk }}$ (Cunningham 1975; Zhang, Cui, \& Chen 1997): $L_{o b s}=g(a, \theta) L_{\text {disk }}$ in which $g(a, \theta)$ is the GR correction factor resulted by GR effects upon the local spectrum depending on both the $\mathrm{BH}$ spin $a$ and the inclination angle $\theta$. For $a=0.94, \theta=61^{\circ}, g(a, \theta)=0.36, l=6.64$. Thus, $\dot{m}=3.27$ from Eq.(1). This means the mass accretion rate $\dot{M}$ is $74.9 M_{\odot} \mathrm{yr}^{-1}$. This value is between the value of $(10.8 \sim 51.4) M_{\odot} \mathrm{yr}^{-1}$ estimated from Gamma-ray observations (Kafatos 1980) and that of $\sim 100 M_{\odot} \mathrm{yr}^{-1}$ estimated in the context of the system of massive binary BHs in the radio band (Roos 1988).

\section{Prediction of the BH mass with radio jet-precession observation}

In the radio band, due to the coupling of magnetic fields in the BH-disk-jet system (e.g., Ustyugova et al. 1995), the observed jet-precession of the galaxy (Rees 1978; Davis, Muxlow, \& Conway 1985; Roos 1988) is the direct reflection of the disk-precession with the same period (Bardeen \& Petterson 1975; Cui, Zhang, \& Chen 1998) induced by the Lense-Thirring (LT) effect. Thus, $p_{\text {jet }}=p$ in which $p_{\text {jet }}$ and $p$ are the precession periods of the jet and the disk, respectively.

Previous authors (e.g., Bardeen, \& Petterson 1975; Sarazin, Begelman, \& Hatchett 1980) demonstrated that LT precessing frequency is $\Omega_{p} \approx \frac{2 J}{r_{p}^{3}}$ at the precession radius $r_{p}$ where $J$ equals to $J_{\text {disk }}=\left(\frac{v_{r}}{v_{\phi}}\right)^{-1} \dot{M} r_{p}^{2}$, the angular momentum of the disk in which $v_{r}$ and $v_{\phi}$ are the velocities of the radial inflow and orbital flow, respectively. Then, $\dot{m}\left(\frac{r_{p}}{300}\right)^{2}=0.3113 \times a \frac{10^{7} v_{r}}{v_{\phi}}$, and $p=1.3233 \times \frac{m}{a}\left(\frac{r_{p}}{300}\right)^{3}$ in which $p=\frac{2 \pi}{\Omega_{p}}$ in unit of $10^{4} \mathrm{yr}$ is the precession period.

Scheuer \& Feiler (1996) found that a disk tends to force the central BH to align with its axis. Besides, if the local LT precession timescale is equal to the one for radial diffusion of the disk warping (or twisting), a radius $R_{\text {warp }}$ will be given simply as the one out to which the alignment of the disk with the BH spin occurs (Pringle 1996). Considering the fact that the outer disk regions $\left(r \geq r_{p}\right)$ cannot be precessed by LT effect but will twist and align the inner disk, together with the central BH, with the outer disk axis (Sarazin, Begelman, \& Hatchett 1980 ), it is clear that $r_{p}=R_{\text {warp }}$. By the expression of $R_{\text {warp }}$ (Natarajan \& Pringle 1998), it is obtained that $\frac{r_{p}}{300}=3.4780 \times a^{0.625} \alpha^{0.75} m^{0.375} \dot{m}^{-0.25}$.

In the middle disk $\left(100 \sim 2000 r_{g}\right), v_{r}$ and $v_{\phi}$ are as follows: $10^{7} v_{r}=$ $1.9212 \alpha^{0.8} m^{-0.6} \dot{m}^{0.4}\left(\frac{r_{p}}{300}\right)^{-0.4}$ and $v_{\phi}=5.7735 \times 10^{-2}\left(\frac{r_{p}}{300}\right)^{-0.5}$ (Novikov, \& Thorne 1973; Bardeen, \& Petterson 1975) in which $\alpha$ is the dimensionless viscous parameter (Shakura, \& Sunyaev 1973).

Above expressions provide following relations:

$$
\left.\begin{array}{l}
m=4.4987 \times a^{-1.3077} \dot{m}^{-0.4615} p^{-0.3846} \\
\alpha=0.9525 \times a^{-1.9} m^{-2.1} \dot{m}^{-0.2} \\
r_{p}=273.2569 \times\left(\frac{a p}{m}\right)^{\frac{1}{3}} \\
v_{r}=1.8547 \times 10^{-8} a^{-1} \dot{m}\left(\frac{r_{p}}{300}\right)^{1.5} \\
v_{\phi}=r_{p}^{-0.5}
\end{array}\right\}
$$

Taking $p=1.5$ (Davis, Muxlow, \& Conway 1985), we obtain for 3C 273: $m=2.4, \alpha=0.134, r_{p}=230.2, v_{r}=4.3 \times 10^{-8}, v_{\phi}=6.6 \times 10^{-2} . m=2.4$ 
means $M_{\mathrm{BH}}=2.4 \times 10^{9} M_{\odot}$, more than Laor's result $\sim 10^{9} M_{\odot}$ (Laor 1998). With this value, the Eddington luminosity is $L_{\mathrm{Edd}}=3.0 \times 10^{47} \mathrm{ergs} \mathrm{s}^{-1}$ and accretion rate is $\dot{M}_{\mathrm{Edd}}=5.5 M_{\odot} \mathrm{yr}^{-1}$. Comparing the two with above corresponding values of $L_{\text {disk }}=8.4 \times 10^{47} \mathrm{ergs} \mathrm{s}^{-1}$ and $\dot{M}=74.9 M_{\odot} \mathrm{yr}^{-1}$, respectively, we are sure of the fact that $3 \mathrm{C} 273$ is a quasar with both the super-Eddington luminosity and the highly super-Eddington accretion rate. Besides, the radial drift velocity $v_{r}$ is so small compared with the azimuthal one $v_{\phi}$ that the ratio of them is only $\sim 10^{-6}$, forming a slowly spiraling accretion to the central body.

In the theory of disk accretion, the nature of the viscosity is the most poorly understood piece (Shapiro, \& Teukolsky 1983). Considering that both the shear amplification and reconnection of a chaotic magnetic field produce the viscosity, Eardley \& Lightman (1975) gave $0.01 \leq \alpha \leq 1$. However, in earlier disk models, $\alpha$ was often simply supposed, in advance, as a constant in theories [e.g., Natarajan, \& Pringle (1998)]. In our work, however, it is revealed that because the set of $\{a, m, \dot{m}\}$ varies with different galaxies, one system should have its own viscosity. This means that $\alpha$ should not be considered as an invariable for different $\mathrm{BH}$ systems. The result of $\alpha=0.134$ for 3C 273 is identical with $\alpha=0.13 \sim 0.14$ presented by Kafatos (1980).

\section{Discussions}

In Section 4, there occurs an assumption: the precession of the large-scale jet is caused by the LT-induced one of the disk with the same frequency. Is this valid?

Bardeen \& Petterson (1975) pointed out that due to the effects of the viscous torques, the disk is almost completely relaxed in the equatorial plane of the $\mathrm{BH}$ in the region of $r>500 r_{g}$ whereas outside the horizon of the $\mathrm{BH}$, bound geodesics of particles in the inner region of the disk extending outside to tens of $r_{g}$ trace out helix-like spherical torus between the minimum and maximum latitudes about the equatorial plane of the central BH. This sketch was verified theoretically and observationally (Wilkins 1972; Chen \& Halpern 1989). Thus, in the range from tens of $r_{g}$ to $500 r_{g}$, there should exists a transition region to adapt the accretion flow from the relatively cold and stable thin-disk morphology sustained by the gas pressure to the thick disk/spherical medium one supported by the radiation pressure [see, e.g., Collin, \& Huré (2001)].

According to López-Corredoira, Betancort-Rijo, \& Beckman (2002), we argue that in the transition region containing the precessing position $\left(r_{p}=230.2\right.$ for 3C 273), an external force should exist to produce a kind of torque which generates the warp of the disk to fit the reorientation of all the different tori and hence constitute a uniform precession of fluid elements at the bottom of the jet to keep the precession upon the entire lobe. Luckily, the magnetic field in the BH-disk-jet system has been considered as the right origins of this force and torque (e.g., Battaner, \& Jiménez-Vicente 1998). Because the magnetic field of the core in $3 \mathrm{C} 273$ is $\sim 0.04 \mathrm{G}$ (Unwin et al. 1985), much greater than the estimated value $1 \mu \mathrm{G}$ to generate observed warps (López-Corredoira, Betancort-Rijo, \& Beckman 2002), it is feasible to work with the assumption.

In general, although it may be too difficult to observe directly the warping of fluids because of the small scales of the warping position (e.g., $r_{p}=230.2 r_{g}$ is only $\sim 0.02 \mathrm{pc}$ ) and the stabilization of the LT precession upon warping insta- 
bility (Pringle 1996), we can not only predict the mass of the central black-hole $(\mathrm{BH})$ in Seyfert(-like) galaxies, but estimate indirectly the position of disk warping and other physical parameters as well, provided that the multiband data are available (Fe line profile in the soft-X ray band, the luminosity in the optical-UV band, and the precession period in the radio band). We hope the method presented in this paper can be used for more Seyfert(-like) galaxies so as to make it possible in the future to study GRMHD coupling in BH-disk-jet systems in Kerr metric with predicted parameters.

Acknowledgments. We thank Prof. J. Wang, NAO, CAS, China, for his crucial support of this work. We emphatically express our gratitude to Prof. J. F. Lu, Xiamen University, China, for his prompt and helpful comments and directions for the original manuscript in Chinese. We also acknowledge the financial support by NAO, CAS.

\section{References}

Bardeen, J. M., \& Petterson, J. A. 1975, ApJ, 195, L65

Bardeen, J. M., Press, W., \& Teukolsky, S. 1972, ApJ, 178, 347

Battaner, E., \& Jiménez-Vicente 1998, A\&A, 332, 809

Chen, K., \& Halpern, J. P. 1989, ApJ, 344, 115

Collin, S., \& Huré, J.-M. 2001, astro-ph/0103303

Courvoisier, T. J.-L., \& Camenzind, M. 1989, A\&A, 224, 10

Cui, W., Zhang, S. N., \& Chen, W. 1998, ApJ, 492, L53

Cunningham, C. T. 1975, ApJ, 202, 788

Czerny, B., Nikolajuk, M., Piasecki, M., \& Kuraszkiewicz, J. 2001, MNRAS, 325,865

Davis, R. J., Muxlow, T. W. B., \& Conway, R. G. 1985, Nature, 318, 343

Dermer, C. D., \& Gehrels, N. 1995, ApJ, 447, 103

Eardley, D. M., \& Lightman, A. P. 1975, ApJ, 200, 187

Fabian, A. C., Iwasawa, K., Reynolds, C. S., \& Young, A. J. 2000, PASP (in press); astro-ph/0004366

Hayashida, K., Miyamoto, S., Kitamoto, S., Negoro, H., \& Inoue, H. 1998, ApJ, 500,642

Kafatos, M. 1980, ApJ, 236, 99

Kriss, G. A., Davidsen, A. F., Zheng, W., \& Lee, G. 1999, ApJ, 527, 683

Laor, A. 1998, ApJ, 505, L83

Leach, C. M., McHardy, I. M., \& Papadakis, I. E. 1995, MNRAS, 272, 221

López-Corredoira, M., Betancort-Rijo, J., \& Beckman, J. E. 2002, preprint (astro-ph/0202156)

Ma, Z. G. 2002, these proceedings

Makishima, K., Maejima, Y., Mitsuda, K., Bradt, H. V., Remillard, R. A., Tuohy, I. R., Hoshi, R., \& Nakagawa, M. 1986, ApJ, 308, 635

Malkan, M. A. 1983, ApJ, 268, 582

Natarajan, P., \& Pringle, J. E. 1998, ApJ, 506, L97 
Novikov, I. D., \& Thorne, K. S. 1973, in Black holes, eds. C. DeWitt \& B. S. DeWitt (New York: Gordon \& Breach), 343

Page, D. N., \& Thorne, K. S. 1974, ApJ, 191, 499

Pringle, J. E. 1996, MNRAS, 281, 357

Rees, M. J. 1978, Nature, 275, 516

Romero, G. E., Chajet, L., Abraham, Z., \& Fan J. H. 2000, A\&A, 360,57

Roos, N. 1988, ApJ, 334, 95

Sarazin, C. L., Begelman, M. C., \& Hatchett, S. P. 1980, ApJ, 238, L129

Scheuer, P. A. G., \& Feiler, R. 1996, MNRAS, 282, 291

Shakura, N. I., \& Sunyaev, R. A. 1973, A\&A, 24, 337

Shapiro, S. L., \& Teukolsky, S. A. 1983, Black Holes, White Dwarfs, and Neutron Stars (New York, Chichester, Brisbane, Toronto, Singapore: John Wiley \& Sons), 403

Unwin, S. C., Cohen, M. H., Biretta, J. A., \& Pearson, T. J. 1985, ApJ, 289, 109

Ustyugova, G. V., Kildoba, A. V., Romanova, A. V., Chechetkin, V. M., \& Lovelace, R. V. E. 1995, ApJ, 439, L39

Wandel, A., \& Mushotzky, R. F. 1986, ApJ, 306, L61

Wilkins, D. C. 1972, Phys.Rev.D, 5, 814

Yaqoob, T., \& Serlemitsos, P. 2000, ApJ, 544, L95

Zhang, S. N., Cui W., \& Chen W. 1997, ApJ, 482, L155 